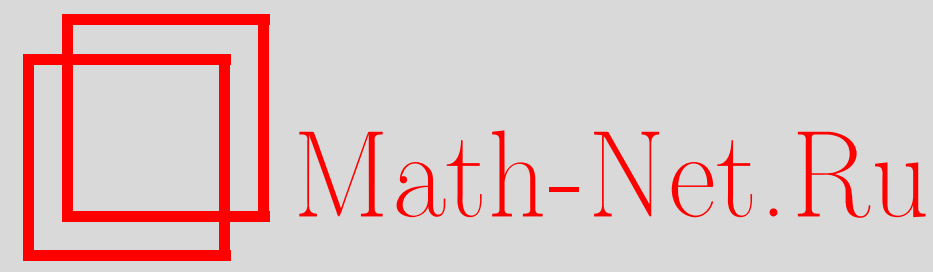

М. С. Сгибнев, Разложение Стоуна для матричной меры восстановления на полуоси, Матем. сб., 2001, том 192, номер 7, 97-106

DOI: https://doi.org/10.4213/sm581

Использование Общероссийского математического портала Math-Net.Ru подразумевает, что вы прочитали и согласны с пользовательским соглашением http: //www.mathnet.ru/rus/agreement

Параметры загрузки:

IP: 54.89 .56 .158

26 апреля 2023 г., 03:34:15 


\title{
М.С. Сгибнев \\ Разложение Стоуна для матричной меры восстановления на полуоси
}

\begin{abstract}
Получено разложение Стоуна для матричной меры восстановления. С помощью данного разложения без труда устанавливается ряд свойств решения системы интегралњых уравнений типа восстановления.

Библиограбфия: 17 названий.
\end{abstract}

\section{§1. Введение}

Пусть $F$ - распределение вероятностей на $\mathbb{R}$ такое, что $\mu \stackrel{\text { def }}{=} \int_{\mathbb{R}} x F(d x) \in$ $(0,+\infty]$ и $m$-кратная свертка $F^{m *}$ при некотором $m \geqslant 1$ содержит ненулевую абсолютно непрерывную компоненту. Обозначим через $F^{0 *}$ атомическую меру массы 1, сосредоточенную в нуле. Стоун [1] показал, что мера восстановления $H \stackrel{\text { def }}{=}$ $\sum_{k=0}^{\infty} F^{m *}$, порожденная распределением $F$, допускает разложение $H=H_{1}+H_{2}$, где $H_{2}$ - конечная мера, а $H_{1}$ - абсолютно непрерьвная мера с ограниченной непрерывной плотностью $h(x)$ такой, что $\lim _{x \rightarrow+\infty} h(x)=1 / \mu$ и $\lim _{x \rightarrow-\infty} h(x)=0$ (см. также [2; теорема 2.6.2]).

Рассмотрим уравнение восстановления

$$
X(t)=g(t)+\int_{\mathbb{R}} X(t-y) F(d y) \stackrel{\text { def }}{=} g(t)+X * F(t),
$$

где $g \in L_{1}(\mathbb{R})$. Асимптотические свойства решения $X(t)$ изучались в [1]-[8]. Очевидно, что $X(t)=g * H(t)+c$ - решение данного уравнения $(c-$ произвольная постоянная). Как отмечено в [6] и показано в [2; теорема 2.6.4], разложение Стоуна $H=H_{1}+H_{2}$ позволяет получить элегантное короткое доказательство следующей ключевой теоремы восстановления: если функция $g \in L_{1}(\mathbb{R})$ ограничена и $\lim _{|x| \rightarrow \infty} g(x)=0$, то

$$
g * H(t) \rightarrow \begin{cases}\mu^{-1} \int_{\mathbb{R}} g(x) d x, & t \rightarrow+\infty \\ 0, & t \rightarrow-\infty\end{cases}
$$

В настоящей работе получен аналог разложения Стоуна для матричной меры восстановления на полуоси. Применение такого разложения позволяет получить

Работа вьполнена при поддержке Российского фонда фундаментальных исследований (грант № 99-01-00504). 
короткое доказательство асимптотических свойств решения системы уравнений типа восстановления при более общих предположениях, чем в [9].

\section{§2. Разложение Стоуна}

Пусть $\mathbf{F}=\left(F_{i j}\right)-$ матрица порядка $n \times n$, элементы которой суть конечные неотрицательные меры, сосредоточенные на $[0, \infty)$. Свертка $\mathbf{F} * \mathbf{G}$ двух таких матриц - это матрица с элементами $(\mathbf{F} * \mathbf{G})_{i j} \stackrel{\text { def }}{=} \sum_{l=1}^{n} F_{i l} * G_{l j}, i, j=1, \ldots, n$.

ОПРЕДЕЛЕНИЕ 1. Матричной мерой восстановления называется матрица мер

$$
\mathbf{H} \stackrel{\text { def }}{=} \sum_{k=0}^{\infty} \mathbf{F}^{k *} ;
$$

здесь $\mathbf{F}^{1 *} \stackrel{\text { def }}{=} \mathbf{F}, \mathbf{F}^{(k+1) *} \stackrel{\text { def }}{=} \mathbf{F}^{k *} * \mathbf{F}=\mathbf{F} * \mathbf{F}^{k *}, \mathbf{F}^{0 *} \stackrel{\text { def }}{=} \delta \mathbf{I}$, где $\delta-$ мера единичной массы, сосредоточенная в нуле, а I - единичная матрица порядка $n \times n$.

Спектральный радиус произвольной числовой матрицы А будем обозначать через $\rho(\mathbf{A})$. По теореме Перрона-Фробениуса [10; теорема 9.2.1] любая неотрицательная неприводимая матрица А имеет положительное собственное значение кратности 1 , равное $\rho(\mathbf{A})$, и существуют положительные правый и левый собственные векторы, соответствующие этому собственному значению.

Нам потребуются утверждения (ii) и (iii) теоремы 2.1 из [11], которые для полноты изложения сформулируем в виде теоремы. С этой целью приведем несколько определений.

ОПРЕДЕЛЕНИЕ 2 [12; гл. XI, §1]. Вешественнозначная функция $g(x), x \geqslant 0$, называется непосредственно интегрируемой по Риману, если при любом $h>0$ ряды

$$
\begin{aligned}
& \underline{\sigma}(h) \stackrel{\text { def }}{=} h \sum_{n=1}^{\infty} \min \{g(x): x \in[(n-1) h, n h]\}, \\
& \bar{\sigma}(h) \stackrel{\text { def }}{=} h \sum_{n=1}^{\infty} \max \{g(x): x \in[(n-1) h, n h]\}
\end{aligned}
$$

сходятся абсолютно и $\lim _{h \rightarrow 0}[\bar{\sigma}(h)-\underline{\sigma}(h)]=0$.

Мера $F$ называется решетчатой с шагом решетки $\lambda$, если она сосредоточена на множестве вида $\{b, b \pm \lambda, b \pm 2 \lambda, \ldots\}$ и $\lambda$ - наибольшее из таких чисел. Если мера $F$ сосредоточена на множестве вида $\{0, \pm \lambda, \pm 2 \lambda, \ldots\}$ и $\lambda$ - наибольшее из таких чисел, то она назьвается арифметической.

ОПРЕДЕЛЕниЕ 3 [13; определение 3.1]. Матрица мер $\mathbf{F}$ называется решетчаmой, если выполнены следующие условия:

$1)$ каждая мера $F_{i j}$ решетчатая с шагом решетки $\lambda_{i j}$, каждая мера $F_{i i}$ арифметическая с шагом решетки $\lambda_{i i}$;

2) все $\lambda_{i j}$ кратны некоторому числу, наибольшее из таких чисел обозначим через $\lambda$;

3) если $a_{i j}, a_{j k}$ и $a_{i k}$ - точки роста функций распределения $F_{i j}(t) \stackrel{\text { def }}{=} F_{i j}([0, t])$, $F_{j k}(t)$ и $F_{i k}(t)$ соответственно, то $a_{i j}+a_{j k}=a_{i k}+m \lambda$. 
Для произвольной вешественнозначной меры $\nu$ определим ее преобразование Лапласа по формуле $\widehat{\nu}(s)=\int_{\mathbb{R}} e^{s x} \nu(d x)$ для тех значений $s \in \mathbb{C}$, при которых данный интеграл сходится абсолютно относительно полной вариации $|\nu|$ меры $\nu$. Условимся: все операции над матрицами мер и вектор-функциями осуществляются поэлементно; например, $\int_{0}^{\infty} x \mathbf{F}(d x) \stackrel{\text { def }}{=}\left(\int_{0}^{\infty} x F_{i j}(d x)\right)$, а если $\mathbf{A}=\left(A_{i j}\right)$ - матрица, элементами которой являются меры, то через $\widehat{\mathbf{A}}(s)$ будем обозначать матрицу, состоящую из преобразований Лапласа $\widehat{A}_{i j}(s)$ элементов матрицы А. Аналогичное соглашение относится и к неравенствам, в которых участвуют матрицы и вектор-функции. Обозначим через $\mathbf{M}(s)$ присоединенную матрицу к $\mathbf{I}-\widehat{\mathbf{F}}(s)$. Положим $\Delta(s) \stackrel{\text { def }}{=}(\mathbf{I}-\widehat{\mathbf{F}}(s))$.

Теорема 1 [11; теорема 2.1 (ii), (iii)]. Пусть $\mathbf{F}$ - нерешетчатая матрица мер такая, что для некоторых $i, j$ мера $F_{i j}$ не сосредоточена в нуле и $\rho[\mathbf{F}(\{0\})]<1$.

(a) Eсли матрица $\mathbf{F}(\infty)$ неприводима и $\rho[\mathbf{F}(\infty)]=1$, то

$$
\mathbf{H}((x, x+h]) \rightarrow-\frac{h \mathbf{M}(0)}{\Delta^{\prime}(0)}, \quad x \rightarrow \infty
$$

для любого $h>0$.

(б) Если матрица $\mathbf{F}(\infty)$ неприводима, существует $\alpha \in \mathbb{R}$ такое, что $\rho[\widehat{\mathbf{F}}(\alpha)]=1$, и функиии $e^{\alpha x} g_{i}(x)$ непосредственно интегрируемь по Риману $i=1, \ldots, n$, mо при $x \rightarrow \infty$

$$
e^{\alpha x} \mathbf{H} * \mathbf{g}(x) \stackrel{\text { def }}{=} e^{\alpha x}\left(\sum_{j=1}^{n} \int_{0}^{x} g_{j}(x-y) H_{i j}(d y)\right) \rightarrow-\frac{\mathbf{M}(\alpha)}{\Delta^{\prime}(\alpha)} \int_{0}^{\infty} e^{\alpha y} \mathbf{g}(y) d y
$$

здесъ $\mathbf{g}(x)=\left(g_{1}(x), \ldots, g_{n}(x)\right)^{T}$.

Абсолютно непрерывную компоненту произвольной неотрицательной меры $\nu$ обозначим через $\nu_{c}$, а ее сингулярную компоненту - через $\nu_{s}: \nu_{s}=\nu-\nu_{c}$; таким образом, под сингулярной компонентой меры здесь понимается сумма ее объчной сингулярной и дискретной компонент. Если $\mathbf{G}=\left(G_{i j}\right)$ - матрица, элементы которой суть неотрицательные меры, то через $\mathbf{G}_{s}$ будем обозначать матрицу $\left(\left(G_{i j}\right)_{s}\right)_{\text {, }}$ элементы которой - сингулярные компоненты соответствующих элементов $\mathbf{G}$.

Сформулируем основной результат работы.

Теорема 2 (разложение Стоуна). Пусть $\mathbf{F}-$ матрица порядка $n \times n$, элементы которой суть конечные неотрицательные меры, сосредоточенные на $[0, \infty)$. Предположим, что матрица $\mathbf{F}(\infty)$ неприводима и $\rho[\mathbf{F}(\infty)]=1$. Виберем левый и правый собственные векторы $\mathbf{l}=\left(l_{1}, \ldots, l_{n}\right)$ u $\mathbf{r}=\left(r_{1}, \ldots, r_{n}\right)^{T}$ с положительными координатами, отвечающие собственному значению 1 матриць $\mathbf{F}(\infty)$. Наконеи, допустим, что $\rho\left[\left(\mathbf{F}^{m *}\right)_{s}(\infty)\right]<1$ при некотором иелом $m \geqslant 1$. Тогда матричная мера восстановления $\mathbf{H}$ допускает разложсение $\mathbf{H}=\mathbf{H}_{1}+\mathbf{H}_{2}$, әде $\mathbf{H}_{2}-$ матрица конечных мер, а $\mathbf{H}_{1}-$ матрица 
абсолютно непрерывных мер с ограниченными непрерывными плотностями $\mathbf{h}(x)=\left(h_{i j}(x)\right)$ такими, что существует предел

$$
\mathbf{h}(+\infty) \stackrel{\text { def }}{=} \lim _{x \rightarrow+\infty} \mathbf{h}(x)=\frac{\mathbf{r l}}{\mathbf{l} \int_{0}^{\infty} x \mathbf{F}(d x) \mathbf{r}}
$$

ДокАЗАТЕльство. В том, что касается общего хода рассуждений, доказательство сформулированной теоремы в известной степени повторяет доказательство для одномерного случая. В то же время при рассмотрении матричного случая возникают специфические трудности, для преодоления которых потребуются дополнительные усилия. Здесь будет использована схема доказательства разложения Стоуна в одномерном случае, предложенная в [2; теорема 2.6.2].

Предположим сначала, что матрицу $\mathbf{F}$ можно представить в виде $\mathbf{F}=\mathbf{F}_{1}+\mathbf{F}_{2}$, где $\mathbf{F}_{1} \neq \mathbf{0}$ - матрица абсолютно непрерывных мер с матрицей плотностей $\mathbf{f}(x)=\left(f_{i j}(x)\right)$, где все $f_{i j}(x)$ - непрерывные функции с компактными носителями: $\operatorname{supp} f_{i j}(x) \subset[c, d], 0 \leqslant c<d<\infty$. Спектральньй радиус $\mathbf{F}_{2}(\infty)$ меньше единицы [14; гл. XIII, $\S 2$, лемма 2], поэтому матричная мера $\mathbf{H}_{2} \stackrel{\text { def }}{=} \sum_{k=0}^{\infty} \mathbf{F}_{2}^{k *}$ конечна: $\mathbf{H}_{2}(\infty)=\left[\mathbf{I}-\mathbf{F}_{2}(\infty)\right]^{-1}<\infty$. По индукции устанавливаем

$$
\mathbf{F}^{k *}=\sum_{j=0}^{k-1} \mathbf{F}^{(k-1-j) *} * \mathbf{F}_{1} * \mathbf{F}_{2}^{j *}+\mathbf{F}_{2}^{k *}, \quad k \geqslant 1
$$

Суммируя по $k$ обе части равенства (2), получаем

$$
\begin{aligned}
\mathbf{H} & =\sum_{k=1}^{\infty} \sum_{j=0}^{k-1} \mathbf{F}^{(k-1-j) *} * \mathbf{F}_{1} * \mathbf{F}_{2}^{j *}+\mathbf{H}_{2} \\
& =\sum_{j=0}^{\infty} \sum_{k=j+1}^{\infty} \mathbf{F}^{(k-1-j) *} * \mathbf{F}_{1} * \mathbf{F}_{2}^{j *}+\mathbf{H}_{2}=\mathbf{H} * \mathbf{F}_{1} * \mathbf{H}_{2}+\mathbf{H}_{2}
\end{aligned}
$$

Покажем, что плотности элементов матрицы $\mathbf{H}_{1} \stackrel{\text { def }}{=} \mathbf{H} * \mathbf{F}_{1} * \mathbf{H}_{2}$ удовлетворяют (1). Поскольку элементы матрицы $\mathbf{f}(x)$ - непосредственно интегрируемые по Риману функции (см. определение 2), имеем согласно утверждению (б) теоремы 1 , в котором полагаем $\alpha=0$,

$$
\mathbf{H} * \mathbf{f}(x) \stackrel{\text { def }}{=}\left(\sum_{l=1}^{n} \int_{0}^{x} f_{l j}(x-y) H_{i l}(d y)\right) \rightarrow-\frac{\mathbf{M}(0)}{\Delta^{\prime}(0)} \mathbf{F}_{1}(\infty), \quad x \rightarrow \infty
$$

причем $\mathbf{M}(0) / \Delta^{\prime}(0) \stackrel{\text { def }}{=} \mathbf{0}$ (нулевая матрица), если хотя бы одно из чисел

$$
\int_{0}^{\infty} x F_{i j}(d x), \quad i, j=1, \ldots, n
$$

бесконечно. Элементы матрицы $\mathbf{H} * \mathbf{f}(x)$ - ограниченные функции, так как значения меры Н ограничены на конечных интервалах постоянной длины; это вытекает 
из утверждения (a) теоремы 1 . Кроме того, элементы $\mathbf{H} * \mathbf{f}(x)$ - непрерывные функции, ибо $\mathbf{f}(x-y)$ непрерывна по $x$ и применима теорема о переходе к пределу под знаком интеграла: мера $\mathbf{H}$ конечна на интервале $[x-d, x-c]$, а вне его $\mathbf{f}(x-y)$ обрашается в нуль. Далее, поскольку матричная мера $\mathbf{H}_{2}$ конечна, при $x \rightarrow \infty$ имеем

$$
\begin{aligned}
\mathbf{h}(x) & \stackrel{\text { def }}{=} \int_{0}^{x} \mathbf{H} * \mathbf{f}(x-y) \mathbf{H}_{2}(d y) \rightarrow-\frac{\mathbf{M}(0)}{\Delta^{\prime}(0)} \mathbf{F}_{1}(\infty) \mathbf{H}_{2}(\infty) \\
& =-\frac{\mathbf{M}(0)}{\Delta^{\prime}(0)} \mathbf{F}_{1}(\infty)\left[\mathbf{I}-\mathbf{F}_{2}(\infty)\right]^{-1} \\
& =-\frac{\mathbf{M}(0)}{\Delta^{\prime}(0)}\left\{\left[\mathbf{I}-\mathbf{F}_{2}(\infty)\right]-[\mathbf{I}-\mathbf{F}(\infty)]\right\}\left[\mathbf{I}-\mathbf{F}_{2}(\infty)\right]^{-1}=-\frac{\mathbf{M}(0)}{\Delta^{\prime}(0)}
\end{aligned}
$$

так как $\mathbf{M}(0)[\mathbf{I}-\mathbf{F}(\infty)]=\Delta(0) \mathbf{I}=\mathbf{0}$. Это завершает доказательство теоремы на первом этапе, если воспользоваться следующей леммой.

Лемма 1. Пусть $\mathbf{F}$ - матрица мер такая, что матрица $\mathbf{F}(\infty)$ неприводима и ее спектральный радиус равен 1. Предположсим, что все әлементы матрицы $\int_{0}^{\infty} x \mathbf{F}(d x)$ конечны. Виберем левый и правый собственные векторь $\mathbf{l}$ и $\mathbf{r}$ с положительными координатами, отвечающие собственному значению 1 матриць $\widehat{\mathbf{F}}(0)$, так, чтобь $\mathbf{l r}=1$. Тогда

$$
-\frac{\mathbf{M}(0)}{\Delta^{\prime}(0)}=\frac{\mathbf{r l}}{\mathbf{1} \int_{0}^{\infty} x \mathbf{F}(d x) \mathbf{r}}
$$

Лемма 1 доказьвается модификацией рассуждения из доказательств лемм 8 и 6 в [15], где равенство (3) установлено в частном случае примитивных стохастических матриц $\mathbf{F}$. А именно: надо воспользоваться тем, что в нашем случае $\sum_{j=0}^{k} \widehat{\mathbf{F}}(q)^{j} / k \rightarrow \mathbf{r l}$ при $k \rightarrow \infty[16 ;$ теорема 8.6.1], и заменить вектор-столбец $\mathbf{e ~ c ~}$ единичными координатами на вектор $\mathbf{r}$.

Очевидно, что справедливость (3) сохранится и в том случае, если хотя бы одно из чисел $\int_{0}^{\infty} x F_{i j}(d x), i, j=1, \ldots, n$, бесконечно (напомним, что в этом случае $\left.-\mathbf{M}(0) / \Delta^{\prime}(0) \stackrel{\text { def }}{=} \mathbf{0}\right)$.

Для завершения доказательства теоремы 1 в общем случае нам потребуется следуюшая лемма.

Лемма 2. Пусть выполнены условия теоремы 1. Тогда найдется целое число $p \geqslant 1$ такое, ито $\mathbf{F}^{p *}=\mathbf{F}_{1}+\mathbf{F}_{2}$, где $\mathbf{F}_{1} \neq \mathbf{0}$ - матрица абсолютно непрерывных неотрицательных мер с непрерьвными плотностями, имеющими компактные носители, а мера восстановления $\mathbf{H}_{2}=\sum_{k=0}^{\infty} \mathbf{F}_{2}^{k *}$ конечна.

ДокАЗАТЕльство ЛЕммы 2. Допустим сначала, что $\mathbf{F}(\infty)$ - примитивная матрица. Тогда $\mathbf{F}(\infty)^{k} \rightarrow \mathbf{r l}>\mathbf{0}$ при $k \rightarrow \infty$ [16; теорема 8.5.1]. Далее, $\left[\left(\mathbf{F}^{m *}\right)_{s}\right]^{k *}(\infty) \rightarrow \mathbf{0}$ при $k \rightarrow \infty\left[16 ;\right.$ следствие 8.1.33]. Поэтому $\left(\mathbf{F}^{k m *}\right)_{c}(\infty) \geqslant$ $\mathbf{F}(\infty)^{k m}-\left[\left(\mathbf{F}^{m *}\right)_{s}\right]^{k *}(\infty)>\mathbf{0}$ при достаточно большом $k$. Следовательно, $\left(\mathbf{F}^{k m *}\right)_{c}$ можно представить в виде $\mathbf{G}_{1}+\mathbf{G}_{2}$, где $\mathbf{G}_{1}$ - матрица абсолютно непрерьвных 
мер с ограниченными плотностями, обращающимися в нуль вне некоторого конечного интервала, причем $\mathbf{G}_{1}(\infty)>\mathbf{0}$. В результате имеем $\mathbf{F}^{p *}=\mathbf{F}_{1}+\mathbf{F}_{2}$, где $p=2 k m$ и $\mathbf{F}_{1}=\mathbf{G}_{1} * \mathbf{G}_{1}$. Матричная мера $\mathbf{F}_{1}$ обладает требуемыми свойствами [17; гл. III, $\S 16$, лемма]. Далее, матрица $\mathbf{F}^{p *}(\infty)$ также примитивна [16; теорема 8.5.2], $\rho\left[\mathbf{F}^{p *}(\infty)\right]=1$ и, следовательно, по лемме 2 из [14; гл. XIII, $\left.\S 2\right]$ $\rho\left[\mathbf{F}_{2}(\infty)\right]<1$, откуда вытекает, что мера $\mathbf{H}_{2}$ конечна.

Если $\mathbf{F}(\infty)$ - импримитивная матрица с индексом импримитивности $d>1$, то, переставляя в случае необходимости одинаково строки и столбцы матрицы $\mathbf{F}(\infty)^{d}$, ее можно разложить на $d$ примитивных матриц, имеющих спектральный радиус 1. Не ограничивая обшности, можно считать, что такая перестановка уже произведена $\left(\mathbf{F}^{(i)}(\infty)\right.$ - примитивные матрицы):

$$
\mathbf{F}(\infty)^{d}=\left(\begin{array}{cccc}
\mathbf{F}^{(1)}(\infty) & 0 & \ldots & 0 \\
0 & \mathbf{F}^{(2)}(\infty) & \ldots & 0 \\
\ldots \ldots & \ldots & \ldots & \ldots \ldots \ldots \ldots \\
0 & 0 & \ldots & \mathbf{F}^{(d)}(\infty)
\end{array}\right)
$$

Покажем, что все блочные матрицы $\mathbf{F}^{(i)}$ суть матрицы того же типа, что и $\mathbf{F}, \mathbf{a}$ именно: найдется целое $q \geqslant 1$ такое, что $\rho\left[\left(\mathbf{F}^{(i)} q *\right)_{s}(\infty)\right]<1 \forall i$. Действительно, из легко проверяемого неравенства $\left(\mathbf{F}^{k m *}\right)_{s}(\infty) \leqslant\left[\left(\mathbf{F}^{m *}\right)_{s}\right]^{k *}(\infty)$ и $[16$; теорема 8.1.18] вытекает

$$
\rho\left[\left(\mathbf{F}^{k m *}\right)_{s}(\infty)\right] \leqslant \rho\left\{\left[\left(\mathbf{F}^{m *}\right)_{s}\right]^{k *}(\infty)\right\}=\left\{\rho\left[\left(\mathbf{F}^{m *}\right)_{s}(\infty)\right]\right\}^{k} .
$$

В силу сделанных предположений выражение, стоящее в правой части, стремится к нулю при $k \rightarrow \infty$. Выбирая $k=l d$ достаточно большим и полагая $q=l m$, имеем

$$
\rho\left[\left(\mathbf{F}^{(i) q *}\right)_{s}(\infty)\right] \leqslant \rho\left[\left(\mathbf{F}^{k m *}\right)_{s}(\infty)\right]<1, \quad i=1, \ldots, d
$$

Производя с $\mathbf{F}^{(i)}(\infty)$ уже известные действия, получаем $\left[\mathbf{F}^{(i)}\right]^{p(i) *}=\mathbf{G}_{1}^{(i)}+\mathbf{G}_{2}^{(i)}$, где $\mathbf{G}_{1}^{(i)}$ - матрица положительных абсолютно непрерывных мер с непрерывными плотностями, обращающимися в нуль вне некоторого конечного интервала, причем $\mathbf{G}_{1}^{(i)}(\infty)>\mathbf{0}$. Положим

$$
p \stackrel{\text { def }}{=} \prod_{i=1}^{d} p(i), \quad q(i) \stackrel{\text { def }}{=} \frac{p}{p(i)}, \quad \mathbf{F}_{1}^{(i)} \stackrel{\text { def }}{=}\left[\mathbf{G}_{1}^{(i)}\right]^{q(i) *} .
$$

Тогда $\mathbf{F}_{1}^{(i)}$ - матрица положительных абсолютно непрерывных мер с непрерывными плотностями, обращающимися в нуль вне некоторого конечного интервала, поскольку свертка конечного числа плотностей с такими свойствами - снова функция того же типа. Кроме того, $\mathbf{F}_{1}^{(i)}(\infty)>\mathbf{0}$ и матричная мера восстановления $\mathbf{H}_{2}^{(i)}$, порожденная матрицей мер $\mathbf{F}_{2}^{(i)}$, конечна. Положим $\mathbf{F}^{p *} \stackrel{\text { def }}{=} \mathbf{F}_{1}+\mathbf{F}_{2}$, где

$$
\mathbf{F}_{1} \stackrel{\text { def }}{=}\left(\begin{array}{cccc}
\mathbf{F}_{1}^{(1)} & 0 & \ldots & 0 \\
0 & \mathbf{F}_{1}^{(2)} & \ldots & 0 \\
\ldots \ldots & \ldots \ldots \ldots & \ldots \\
0 & 0 & \ldots & \mathbf{F}_{1}^{(d)}
\end{array}\right)
$$


Поскольку у блочно-диагональной матрицы $\mathbf{H}_{2}=\operatorname{diag}\left\{\mathbf{H}_{2}^{(1)}, \ldots, \mathbf{H}_{2}^{(d)}\right\}$ все блоки $\mathbf{H}_{2}^{(i)}$ - конечные меры, отсюда вытекает $\mathbf{H}_{2}(\infty)<\infty$. Лемма 2 доказана.

Завершим доказательство теоремы 2. Положим $\mathbf{H}^{(0)} \stackrel{\text { def }}{=} \sum_{k=0}^{\infty} \mathbf{F}^{k p *}$ и $\mathbf{H}^{(j)} \stackrel{\text { def }}{=}$ $\mathbf{F}^{j *} * \mathbf{H}^{(0)}, j=1, \ldots, p-1$. Обозначим через $\mathbf{M}_{p}(s)$ присоединенную матрицу к $\mathbf{I}-\widehat{\mathbf{F}}(s)^{p}$. Положим $\Delta_{p}(s) \stackrel{\text { def }}{=} \operatorname{det}\left(\mathbf{I}-\widehat{\mathbf{F}}(s)^{p}\right)$. Согласно уже доказанному на первом этапе $\mathbf{H}^{(0)}=\mathbf{H}_{1}^{(0)}+\mathbf{H}_{2}^{(0)}$, где $\mathbf{H}_{2}^{(0)}(\infty)<\infty$, а $\mathbf{H}_{1}^{(0)}$ - матрица неотрицательных абсолютно непрерывных мер с ограниченными непрерывными плотностями $\mathbf{h}^{(0)}(x)=\left(h_{i j}^{(0)}(x)\right)$ такими, что

$$
\lim _{x \rightarrow \infty} h_{i j}^{(0)}(x)=-\lim _{s \rightarrow 0-} \frac{\mathbf{M}_{p}(s)}{\Delta_{p}(s) / s}, \quad i, j=1, \ldots, n .
$$

Положим $\mathbf{H}_{i} \stackrel{\text { def }}{=} \sum_{j=0}^{p-1} \mathbf{F}^{j *} * \mathbf{H}_{i}^{(0)}, i=1,2$. Тогда $\mathbf{H}=\mathbf{H}_{1}+\mathbf{H}_{2}, \mathbf{H}_{2}(\infty)<\infty$ и матричная мера $\mathbf{H}_{1}$ обладает требуемыми свойствами. Действительно, при $x \rightarrow \infty$ имеем

$$
\begin{aligned}
\mathbf{h}(x) & \stackrel{\text { def }}{=} \sum_{j=0}^{p-1} \mathbf{F}^{j *} * \mathbf{h}^{(0)}(x) \rightarrow-\sum_{j=0}^{p-1} \widehat{\mathbf{F}}(0)^{j} \lim _{s \rightarrow 0-} \frac{\mathbf{M}_{p}(s)}{\Delta_{p}(s) / s} \\
& =-\lim _{s \rightarrow 0-} \sum_{j=0}^{p-1} \frac{\widehat{\mathbf{F}}(s)^{j} \mathbf{M}_{p}(s)}{\Delta_{p}(s) / s}=-\lim _{s \rightarrow 0-} \frac{[\mathbf{I}-\widehat{\mathbf{F}}(s)]^{-1}\left[\mathbf{I}-\widehat{\mathbf{F}}(s)^{p}\right] \mathbf{M}_{p}(s)}{\Delta_{p}(s) / s} \\
& =-\lim _{s \rightarrow 0-}[\mathbf{I}-\widehat{\mathbf{F}}(s)]^{-1} s=-\lim _{s \rightarrow 0-} \frac{\mathbf{M}(s)}{\Delta(s) / s}=-\frac{\mathbf{M}(0)}{\Delta^{\prime}(0)}=\frac{\mathbf{r l}}{\mathbf{1} \int_{0}^{\infty} x \mathbf{F}(d x) \mathbf{r}}
\end{aligned}
$$

Теорема 2 доказана.

\section{§3. Система интегральных уравнений}

Рассмотрим систему интегральных уравнений типа восстановления

$$
Z_{i}(x)=g_{i}(x)+\sum_{j=1}^{n} \int_{0}^{x} Z_{j}(x-u) F_{i j}(d u), \quad i=1, \ldots, n,
$$

где $g_{i} \in L_{1}(0, \infty), i=1, \ldots, n$, а $\mathbf{F}=\left(F_{i j}\right)$ - матрица неотрицательных конечных мер, сосредоточенных на $[0, \infty)$. В матричной форме система (4) принимает вид $\mathbf{Z}(x)=\mathbf{g}(x)+\mathbf{F} * \mathbf{Z}(x)$, где элемент $(\mathbf{F} * \mathbf{Z}(x))_{i}$ по определению равен сумме в правой части (4). Пусть матрица $\mathbf{F}(\infty)$ неприводима и $\rho[\mathbf{F}(\infty)]=1$. Обозначим $M_{l} \stackrel{\text { def }}{=}\left\{f \in L_{\infty}(0, \infty): \exists f(+\infty)\right\}, M_{0} \stackrel{\text { def }}{=}\left\{f \in M_{l}: f(+\infty)=0\right\}, C_{l} \stackrel{\text { def }}{=}$ $\{f \in C(0, \infty): \exists f(+\infty)\}, C_{0} \stackrel{\text { def }}{=}\left\{f \in C_{l}: F(+\infty)=0\right\}$. Предел $f(+\infty)$ в $M_{l}$ понимается в следующем смысле: в классе эквивалентных функций найдется представитель $\widetilde{f}$ такой, что сушествует обычный предел $\widetilde{f}(+\infty)=\lim _{x \rightarrow+\infty} \widetilde{f}(x)$ и $f(+\infty) \stackrel{\text { def }}{=} \widetilde{f}(+\infty)$. 
Система уравнений (4) рассматривалась в [9], где предполагалось, что все меры $F_{i j}$ абсолютно непрерывны, т.е. $\mathbf{F}(d x)=\mathbf{f}(x) d x$, где $\mathbf{f} \in L_{1}(0, \infty)$. В теореме 5.1 из [9] утверждается, что при сделанных выше предположениях решение системы (4) имеет вид

$$
\mathbf{Z}(x)=\frac{\mathbf{r} \mathbf{l} \int_{0}^{\infty} \mathbf{g}(y) d y}{\mathbf{l} \int_{0}^{\infty} y \mathbf{f}(y) d y \mathbf{r}}+\boldsymbol{\rho}_{0}(x)+\boldsymbol{\psi}(x)
$$

где $\boldsymbol{\rho}_{0} \in C_{0}(0, \infty), \boldsymbol{\psi} \in L_{1}(0, \infty)$; если предположить дополнительно, что $\mathbf{g} \in M_{0}$, то тогда и $\boldsymbol{\psi} \in M_{0}$.

Теперь применим теорему 2 к исследованию асимптотических свойств решения системы (4), не предполагая абсолютной непрерьвности элементов матрицы F.

ТеОРема 3. Пусть $\mathbf{F}-$ матрица порядка $n \times n$, әлементы которой суть конечные неотричательные мерь, сосредоточенные на $[0, \infty)$. Предположим, что матрица $\mathbf{F}(\infty)$ неприводима и $\rho[\mathbf{F}(\infty)]=1$. Выберем левый и правый собственные векторы $\mathbf{l}=\left(l_{1}, \ldots, l_{n}\right)$ u $\mathbf{r}=\left(r_{1}, \ldots, r_{n}\right)^{T}$ с положительными координатами, отвечающие собственному значению 1 матрицы $\mathbf{F}(\infty)$. Допустим, что $\rho\left[\left(\mathbf{F}^{m *}\right)_{s}(\infty)\right]<1$ при некотором иелом $m \geqslant 1$. Пусть $\mathbf{g} \in L_{1}(0, \infty)$ - вектор-функиия. Тогда существует решение $\mathbf{Z}=\mathbf{Z}_{1}+\mathbf{Z}_{2}$ системы (4) такое, что $\mathbf{Z}_{1} \in C_{l}, \mathbf{Z}_{2} \in L_{1}(0, \infty) u$

$$
\mathbf{Z}_{1}(+\infty)=\frac{\mathbf{r l} \int_{0}^{\infty} \mathbf{g}(x) d x}{\mathbf{l} \int_{0}^{\infty} x \mathbf{F}(d x) \mathbf{r}}
$$

Если дополнительно предположить $\mathbf{g} \in M_{0}\left(C_{0}\right)$, mо $\mathbf{Z}_{2} \in M_{0}\left(C_{0}\right)$, так что $\mathbf{Z} \in M_{l}\left(C_{l}\right)$.

ДокАзАТЕЛЬСтво. Не ограничивая общности, можно считать $\mathbf{g}(x) \geqslant 0$ п.в. Очевидно, что $\mathbf{Z}(x) \stackrel{\text { def }}{=} \mathbf{H} * \mathbf{g}(x)$ - решение системы (4); здесь $\mathbf{H}$ - матричная мера восстановления, порожденная матрицей $\mathbf{F}$. Далее, $\mathbf{Z}(x)<\infty$ п.в. Действительно, рассмотрим конечную вектор-меру $\mathbf{G}$ с плотностью $\mathbf{g}(x)$. Тогда вектор-мера $\mathbf{H} * \mathbf{G} \sigma$-конечна, поскольку (как уже отмечалось выше) $\sup _{x \geqslant 0} \mathbf{H}([x, x+h])<\infty$ $\forall h>0$. Так как $\mathbf{Z}(x)$ - плотность вектор-меры $\mathbf{H} * \mathbf{G}, \mathbf{Z}(x)<\infty$ п.в.

Пусть $\mathbf{H}=\mathbf{H}_{1}+\mathbf{H}_{2}-$ разложение Стоуна, доставляемое теоремой 1 . Положим $\mathbf{Z}_{i} \stackrel{\text { def }}{=} \mathbf{H}_{i} * \mathbf{g}, i=1,2$. По теореме Фубини $\mathbf{Z}_{2} \in L_{1}(0, \infty)$. Равенство для $\mathbf{Z}_{1}(+\infty)$ устанавливается по аналогии с одномерным случаем [2; теорема 2.6.4 (a)]. Очевидно, что $\mathbf{Z}_{1} \in C(0, \infty)$. Далее,

$$
\begin{aligned}
\mathbf{Z}_{1}(x) & =\frac{\mathbf{r l}}{\mathbf{l} \int_{0}^{\infty} x \mathbf{F}(d x) \mathbf{r}} \int_{0}^{x} \mathbf{g}(y) d y \\
& +\int_{0}^{x}\left[\mathbf{h}(x-y)-\frac{\mathbf{r l}}{\mathbf{l} \int_{0}^{\infty} x \mathbf{F}(d x) \mathbf{r}} \mathbf{1}_{(0, \infty)}(x-y)\right] \mathbf{g}(y) d y \stackrel{\text { def }}{=} \mathbf{Z}_{3}(x)+\mathbf{Z}_{4}(x) ;
\end{aligned}
$$


здесь $\mathbf{1}_{(0, \infty)}(x)$ - индикатор множества $(0, \infty)$. Имеем

$$
\mathbf{Z}_{3}(x) \rightarrow \frac{\mathbf{r l} \int_{0}^{\infty} \mathbf{g}(x) d x}{\mathbf{1} \int_{0}^{\infty} x \mathbf{F}(d x) \mathbf{r}}, \quad x \rightarrow \infty
$$

и по теореме о мажорируемой сходимости $\mathbf{Z}_{4}(x) \rightarrow 0$ при $x \rightarrow \infty$. Допустим, что $\mathbf{g} \in M_{0}$. Тогда $\exists \widetilde{\mathbf{g}}=\left(\widetilde{g}_{1}, \ldots, \widetilde{g}_{n}\right)^{T} \in M_{0}$ такое, что $\widetilde{\mathbf{g}}(x)=\mathbf{g}(x)$ п.в., $\left|\widetilde{g}_{i}(x)\right| \leqslant$ $\left\|g_{i}\right\|_{\infty} \forall i, x$ и $\exists \widetilde{\mathbf{g}}(+\infty)=0$ в обычном смысле. Имеем

$$
\mathbf{Z}_{2}(x)=\mathbf{H}_{2} * \widetilde{\mathbf{g}}(x)+\mathbf{H}_{2} *[\mathbf{g}-\widetilde{\mathbf{g}}](x) \stackrel{\text { def }}{=} \mathbf{Y}(x)+\left[\mathbf{Z}_{2}(x)-\mathbf{Y}(x)\right]
$$

По теореме о мажорируемости сходимости $\exists \mathbf{Y}(+\infty)=\mathbf{H}_{2}(+\infty) \widetilde{\mathbf{g}}(+\infty)=0$. Таким образом, $\mathbf{Y} \in M_{0}$. По теореме Фубини $\int_{0}^{\infty}\left|\left(\mathbf{Z}_{2}(x)\right)_{i}-(\mathbf{Y}(x))_{i}\right| d x=0 \forall i$. Следовательно, $\mathbf{Z}_{2}(x)=\mathbf{Y}(x)$ п.в. Это означает $\mathbf{Z}_{2} \in M_{0}$. Теорема 3 доказана.

ЗАмЕчАнИЕ 1 . В множестве вектор-функций $\mathbf{f}(x), x \geqslant 0$, для которых определено преобразование Лапласа $\widehat{\mathbf{f}}(s)=\int_{0}^{\infty} e^{s x} \mathbf{f}(x) d x$ при $\operatorname{Re} s<\alpha \leqslant 0$, решение $\mathbf{H} * \mathbf{g}(x)$ системы $(4)$ единственно с точностью до эквивалентности п.в. Это вытекает из очевидных равенств $\widehat{\mathbf{Z}}(s)=[\mathbf{I}-\widehat{\mathbf{F}}(s)]^{-1} \widehat{\mathbf{g}}(s)=\widehat{\mathbf{H}}(s) \widehat{\mathbf{g}}(s)$.

Автор благодарит рецензента за внимательное прочтение рукописи и ценные замечания.

\section{Список литературы}

1. Stone C. On absolutely continuous components and renewal theory // Ann. Math. Stat. 1966. V. 37. P. 271-275.

2. Alsmeyer G. Erneuerungstheorie. Stuttgart: Teubner, 1991.

3. Smith W. L. Regenerative stochastic processes // Proc. Roy. Soc. London Ser. A. 1955. V. 232. P. 6-31.

4. Smith W. L. Remarks on the paper "Regenerative stochastic processes" // Proc. Roy. Soc. London Ser. A. 1960. V. 256. P. 296-301.

5. Schäl M. Über Lösungen einer Erneuerungsgleichung // Abh. Math. Sem. Univ. Hamburg. 1971. V. 36. P. 89-98.

6. Arjas E., Nummelin E., Tweedie R. L. Uniform limit theorems for non-singular renewal and Markov renewal processes // J. Appl. Probab. 1978. V. 15. P. 112-125.

7. Енгибарян Н. Б. Уравнения восстановления на полуоси // Изв. РАН. Сер. матем. 1999. T. 63. № 1. C. 61-76.

8. Yengibarian N. B. Renewal equation on the whole line // Stochastic Process. Appl. 2000. V. 85. P. 237-247.

9. Енгибарян Н.Б. Теоремы восстановления для системы интегральных уравнений // Матем. сб. 1998. Т. 189. № 12. С. 59-72.

10. Ланкастер П. Теория матриц. М.: Наука, 1982.

11. Crump K.S. On systems of renewal equations: the reducible case // J. Math. Anal. Appl. 1970. V. 31. Р. 517-528.

12. Феллер В. Введение в теорию вероятностей и ее приложения. Т. 2. М.: Наука, 1967.

13. Crump K.S. On systems of renewal equations // J. Math. Anal. Appl. 1970. V. 30. P. $425-434$.

14. Гантмахер Ф. Р. Теория матриц. М.: Наука, 1967. 
15. Jelenković P. R., Lazar A. A. Subexponential asymptotics of a Markov-modulated random walk with queueing applications // J. Appl. Probab. 1998. V. 35. P. 325-347.

16. Хорн Р., Джсонсон Ч. Матричный анализ. М.: Мир, 1989.

17. Гельфанд И. М., Райков Д. А., Шилов Г. Е. Коммутативные нормированные кольца. М.: Физматгиз, 1960.

Институт математики им. С. Л. Соболева СО РАН, г. Новосибирск 